\title{
A Review on Renewable Energy Sources in Microgrid
}

\author{
Navreet Kaur Grewal \\ Research Scholar \\ Guru Nanak Dev Engineering College, \\ Ludhiana
}

\author{
Rupinderjit Singh \\ Associate Professor \\ Guru Nanak Dev Engineering College, \\ Ludhiana
}

\begin{abstract}
The utilization of RES to address electrical energy requirements is gaining traction as a potential solution to this issue of electrical energy shortages. The use of renewable energy sources in electrical generation systems is done in a variety of ways, including in microgrid systems. Microgrid technologies have massive benefits for both the user as well as the electric utility provider. There has been a lot of microgrid development in numerous nations since micro grids have a lot of benefits, such as higher power quality and being more ecologically friendly. Power generation, microgrid architecture, power electronics, control systems, and security devices are all areas of microgrid innovation. In this paper, it is demonstrated that persistence as well as artificial neural networks have certain qualities that make them useful to investigators of controllers at various levels of control that micro grids must have in order to be cost-effective, productive, as well as capable of meeting energy power quality and quantity requirements.
\end{abstract}

\section{Keywords: Renewable energy sources, Microgrid, persistence, Artificial Neural Network}

\section{INTRODUCTION}

There is an increasing need to develop an energy system that is environmentally friendly, cost-effective, and dependable. Distributed power generation based on renewable energy is one part of the answer required to attain that goal[1]. If specific characteristics and needs are met, well-defined areas of distributed energy generation could be deemed microgrids (MGs); but, various challenges must be resolved before large-scale penetration of renewable energy-based MGs into the current electrical network can occur.

Thus, the structure of this article is as follows: section II describes introduction of microgrid and Section III and IV describes a persistence and ANN technique \& Section V gives the survey of previous work Section VI wraps up the paper.

\section{MICROGRIDS}

Various groups have presented the idea of MG, with varying definitions, but the three distinguishing characteristics[2]. A microgrid is a self-contained independent entity that has a "plug-and-play" capability for any distributed energy supply, allowing it to meet the demands of local loads. MGs can become complicated systems with variable degrees of uncertainty as well as the necessity for complex controllers as a result of all of these qualities. The following are some of the important controls that must be considered for an MG:

- Load tracking: Because there are many generators in a traditional bulk power system that store energy in their rotating masses, inertia impacts to the energy balance quickly when a load changes, resulting in a slight reduction in system frequency. MGs, on the other hand, have little or no parts that create inertia, hence control mechanisms to successfully track loads despite abrupt load changes should be implemented. 
- Control of real and reactive power: In traditional electrical networks, synchronous generators provide the majority of real and reactive power. Supposing a small power angle, the output real and reactive powers are roughly decoupled and could be controlled independently; this concept of independent excitation has been extensively researched [3-4]. Power coupling occurs when dispersed energy sources are linked to low-voltage MGs, which can degrade the system's dynamic reactivity and reliability.

- Voltage regulation: In a traditional power grid, the impedance among conventional generation units is sufficient to avoid reactive currents from circulating. However, because MGs often comprise of a variety of distributed energy resources, voltage or reactive power oscillations may occur if the local controllers do not have voltage regulation control.

- Frequency rules: Frequency management in the traditional power grid is based on active power dynamic. In the case of MGs, each local controller's power-frequency droop characteristics should work in accordance with a reference set by the energy management.

- Load sharing: The loads must be shared among distributed energy resources, while emergency controls that require fast load sharing or shedding should be applied without communication.

- Protection: In both grid linked $\&$ islanded modes, many security measures need be in place to detect losses, communication faults, as well as effectively respond to fault currents. Fault currents in grid-connected or islanded MG systems are notably different, as per the literature [5]. As a result, the security of MG networks necessitates measures that vary from those utilized in traditional distribution networks.

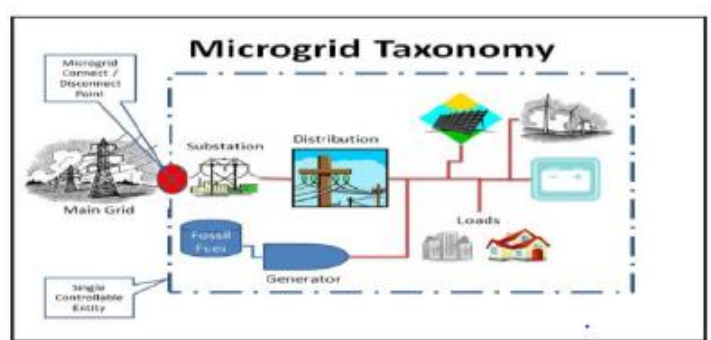

Fig.1. Elementary Microgrid Architecture.[6]

\section{Persistence Technique}

A persistence approach is a easiest and most economical approach to forecast the wind speed. This technique is depend on the assumptions of a high correlation among the present and future wind speed values. If the calculated wind speed at time $(\mathrm{t})$ is $\mathrm{V}(\mathrm{t})$ and $\mathrm{P}(\mathrm{t})$, then the forecast wind speed at $\mathrm{t}+\Delta \mathrm{t}$ could be formulated as a linear equation and is as follows[7]:

$$
V(t+\Delta t)=V(t)
$$

$$
P(t+\Delta t)=P(t)
$$

The above linear equation shows that it is assumed that wind speed at time ' $t+\Delta t^{\prime}$ will be same as it was at time ' $t$ '. This approach is more accurate than most of the physical and statistical methods for very short-term wind speed forecasting. Hence, for latest forecasting approaches should be tested against persistence technique in order to validate the performance of this strategy. Limitation of the persistence technique is that if the forecasting lead time gets enhanced the accuracy of this technique gets decreased.

\section{Artificial Neural Network (ANN) Models}

The Artificial Neural Network is a paradigm of analysis essentially modeled after the brain's vast particular sentence. The nonlinear and complex problem is discussed by the ANN in regards to standards (or) prediction. ANN has the 
ability to conduct nonlinear and complex modeling without a previous assessment of the connection among input and variables.ANN is qualified to know the correlation between various data and output wind speed on the basis of historical wind speed calculation data collected over even a long period of time.

ANN possess better self learning ability (so that it learns the connection among inputs and outputs of any mathematical formulation), adaptability, real-time operation, fault tolerance ability, and cost effective. Several kinds of ANN approaches are feed-forward (BPN, MLP, RBFN), Feedback (ELMAN, Recurrent), Support Vector Machine (SVM), ADALINE, Probability Neural Network (PNN), and so on. Limitation of ANN method contains falling into local minimum, slow convergence, difficult to confirm the structure of network (or) device . In spite of this limitation, ANN approach outperforms than time series technique for all time scale.

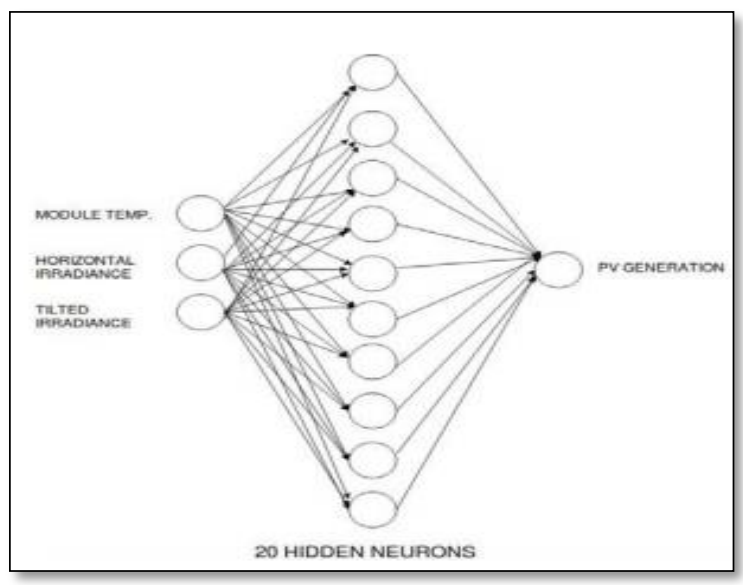

Figure 2:Architecture of Neural Network[14]

\section{LITERATURE SURVEY}

Sanchez et al.,[8] A real-time simulation of an NSML controller for a microgrid linked to a grid was implemented. To monitor the active and reactive forces that are injected into the grid, the suggested local controller for each device is used. An RHONN identification is used to base each suggested local controller. The neural identifiers represent the corresponding nonlinear dynamics and enable disturbances induced by parameter variance and/or irregular situations of the grid to be rejected by the operator. The findings of real-time simulation show the effectiveness of the suggested scheme in achieving trajectory monitoring of DER communication system even in the existence of grid disturbances.

Yuan et al.,[9]Based on modified particle swarm optimization, renewable energy sources are forecast to use the BP neural network (MPSO-BP). The updated method is faster in both search ability and speed contrasted to the standard method. After forecasting, The optimal platform is developed with the goals of total required cost and minimum loss of power. By organizing all sorts of DGs, the performance is optimized. The simulations outcomes confirmed the efficacy of the suggested design in hybrid microgrid multi-objective optimization. The hybrid microgrid was able to realize the optimized level of service via the energy management dispatch.

Usman et al.,[10] introduces an MG energy management system (M-EMS) for grid-connected photovoltaic (PV) and battery energy storage system (BESS) based hybrid MG. The suggested M-EMS comprises of two components forecasting and optimization. The forecasting component is responsible for forecasting solar irradiance, heat and load demand, while the optimization module conducts optimal day-ahead planning of power generation and transmission demand in a grid-connected MG for economical operation. The suggested M-EMS for grid-connected hybrid PV-BESS MG is validated utilizing MATLAB/Simulink. Simulation result shows the productivity and effectiveness of the suggested approach for understudy case.

Vishnu et al.,[11]introduced an energy management device for the microgrid current situation at Wroclaw University of Science and Technology. It has 3 parts: a forecasting structure, an optimization and an enhanced electrical vehicle charger adapter as a different load for the scheme. The 
forecasting framework is based on a deep teaching method using an architecture based on Auto encoder-based LSTM. And uses a numerical analysis of its productivity over many runs and recognizes reliability and time sequence concerns while also gathering evidence for its implementation. A MIDACO - MATPOWER coupled optimal solution is used as the optimization technique for energy management which seeks to harness the velocity of MATPOWER and the analysis tools of Mixed Integer Distributed Ant Colony Optimization (MIDACO) in choosing an effective global minimum solution. The aim of the problem is to reduce the supply of power from the main grid resulting in enhanced self-sufficiency. Eventually, an optimized electrical vehicle charging station method to maximize the renewable energy utilization within the facility is integrated into the same.

Ruicheng et al.,[12] an modified probabilistic load forecasting framework is suggested to adapt to the effect of renewable energy. Firstly, probabilistic residual forecast design is established to capture the uncertainty of load. Then, implemented with the help of forecast mixture and variable selection, the enhanced point load forecast system is generated. Renewable power is regarded in both designs to analyze the consideration among load and renewable energy and modify the outcome. The numerical results of the study demonstrate that the designed method helps improve the predictive power and has greater adaptability to increasing capacity of renewable energy.

Gill et al.,[13]an optimized Fuzzy Interference Method (FIS) for short-term forecasting intervals, which takes time, weather, temperature and relative humidity into account, is suggested. It aims to limit energy sources' maintenance costs. In addition, to measure the percent error between actual and projected load, FIS, particle swarm optimization (PSO) and Catfish algorithms have been implement to these variables. The simulation outcomes indicated that for FIS, PSO and Catfish protocols, the percent error among real and forecast load is below \pm 3 percent, \pm 5 percent and \pm 6 percent.
Also, the suggested approach is consistent to M. And Rizwan et. al [14] where the relative error was 6 percent . The findings have shown that the suggested framework has considerable potential for practical use in power devices.

Li et al.,[15] A new technique of forecasting power load using the feedback neural network with the feature of input delay is suggested in this study in addition to the challenges in conventional forecasting mathematical designs that lack the capacity to self-learn, self-adapt and have poor reliability of the forecast system. The features of the design of the Elman neural network are examined, the Elman neural network experimental framework is designed, and then tested in the power grid with actual data. The findings demonstrate that this model-based power grid load forecasting has good precision and has good sensitivity and learning potential for power grid load.

Imani et al.,[16]For short-term electrical load forecasting, the implementation of a long short-term memory (LSTM) network with support vector regression (SVR) is suggested in this study. For load forecasting, three separate situations are implemented and evaluated. However, in both feature extraction and forecasting, LSTM could be used alone, but the information obtained by LSTM could be used for forecasting as an SVR input. The test results reveal that successful forecasting outcomes are obtained by using load characteristics and temperature characteristics derived by specific LSTM networks in addition to the original load and temperature measurement parameters of the last 24 hours.

Wensheng et al.,[17] proposed a local Integrated Energy Load Prognostic Approach depend on the CNN-LSTM design with consumer energy label distinction. Differential user-based analysis might use actions and allow the person use the tag, and then climate conditions like time period 3 users could use the label as input data depending on the energy form, CNN - LSTM model for load forecasting and verification, in which CNN derives successful input images and LSTM is great at storing time series data. The CNN 
mark distinction - LSTM design has strong performance at the local level of detailed power load forecasting, as per user-based outcomes.

Foladi et al.,[18] a smart charging scheme is proposed for PHEVs that could decrease the energy drawn from the major grid, and this minimizes the dependence of MGs on the major grid by largely the use of RERs/DERs. Two cases are performed on the enhanced version of the IEEE 33-bus test device to verify the effectiveness of the suggested power management method for distinct penetration levels of PHEVs, and the outcomes of the suggested method are contrasted with the outcomes of other, previously reported methods. The suggested outcomes from off-line digital time-domain simulations and software verification represent that the energy exchanged among the MG and the major grid to charge PHEVs is minimized since the RER/DER generation is enhanced. Therefore, the acquired outcomes reveal the superiority of the suggested power management method over other, previously reported methods.

\section{CONCLUSION}

Microgrids are a form of primary electricity systems that could be used to satisfy future electricity demands. The microgrid framework comprises of several power generators, some of which can be renewable. Because the microgrid device is self-contained, it necessitates a sophisticated control system to ensure proper operation. MGs can help to cut down on energy transmission and distribution losses. MGs can aid in the improvement of safety and dependability. This review provides an introduction to MGs and a literature review on improving energy efficiency via the use of various technologies, as well as a brief overview of persistence $\& \mathrm{NN}$ architectures that have been used in the field of MG control to enhance power system reliability.

\section{REFERENCES}

[1] Lopez-Garcia, T. B., Coronado-Mendoza, A., \& Domínguez-Navarro, J. A., "Artificial neural networks in microgrids: A review", Engineering Applications of Artificial Intelligence, Vol. 95,,2020.

[2] Rehtanz, C., "Autonomous Systems and Intelligent Agents in Power System Control and Operation", first ed. Springer-Verlag Berlin Heidelberg, Berlin,2003.

[3] Akagi, H., Kanazawa, Y., Nabae, A., "Instantaneous reactive power compensators comprising switching devices without energy storage components", IEEE Trans. Ind. Appl. IA-20 Vol. 3, pp. 625-630,1984.

[4] Machowski, J., Robak, S., Bialek, J., Bumby, J., AbiSamra, N., "Decentralized stability-enhancing control of synchronous generator", IEEE Trans. Power Syst., Vol. 15, No. 4, pp. 1336-1344,2000.

[5] Monfared, M., Golestan, S., "Control strategies for single-phase grid integration of small-scale renewable energy sources: A review", Renew. Sustain. Energy Rev., Vol. 16, No. 7, pp.4982-4993,2012.

[6] Bayindir R, Hossain E, Kabalci E, Perez R., "A comprehensive study on microgrid technology", Int J Renew Energy Res , Vol.4, pp. 1094-107,2014.

[7] Et-Taoussi, M., Ouadi, H., Chakir, H., "Hybrid optimal management of active and reactive power flow in a smart microgrid with photovoltaic generation", Microsystem Technologies, 2018 .

[8]Larbi Djilali , Edgar N. Sanchez , Fernando OrnelasTellez, Alberto Avalos, and Mohammed Belkheiri, "Improving Microgrid Low-Voltage Ride-Through Capacity Using Neural Control”, IEEE Systems Journal,2019.

[9] Yuan, B., Chen, A., Du, C., \& Zhang, C. , “ Hybrid AC/DC microgrid energy management based on renewable 
energy sources forecasting", 36th Chinese Control Conference (CCC),2017.

[10] Usman Bashir Tayab, Fuwen Yang, Mohamed ElHendawi, and Junwei Lu, "Energy Management System for a Grid-Connected Microgrid with Photovoltaic and Battery Energy Storage System”, 2018 Australian \& New Zealand Control Conference (ANZCC),2018.

[11] Vishnu S., Przemyslaw J., Josep M. , “Microgrid Energy Management System With Embedded Deep Learning Forecaster and Combined Optimizer",IEEE, Vol. 8, 2020.

[12]Ruicheng Zheng, Zhijian Jin , "Probabilistic Load Forecasting with High Penetration of Renewable Energy Based on Variable Selection and Residual Modeling ", IEEE,2019.

[13] Gill, B. B., Singh, M., \& Kakkar, A. , "Improving Load Forecasting and Renewable Energy Management for Green Computing using FIS, PSO and Catfish", IEEE 10th Annual Information Technology, Electronics and Mobile Communication Conference (IEMCON),2019.

[14]M. Rizwan, Majid Jamil, D. P. Kothari, “ Generalized Neural Network Approach for Global Solar Energy Estimation in India", IEEE Transactions on Sustainable Energy, pp.576-584,2012.

[15] Li, D., Wang, H., Zhang, Y., \& Fang, C. , “ Power Grid Load State Information Perception Forecasting Technology for Battery Energy Storage System Based on Elman Neural Network", IEEE 3rd Information Technology, Networking, Electronic and Automation Control Conference (ITNEC), 2019.

[16] Imani, M. , “ Long Short-Term Memory Network and Support Vector Regression for Electrical Load Forecasting”, International Conference on Power Generation Systems and Renewable Energy Technologies (PGSRET),2019.
[17] Li Wensheng, Wang Yanshuo, Wu Kuihua, “A RegionLevel Integrated Energy Load Forecasting Method Based on CNN-LSTM Model with User Energy Label Differentiation",IEEE,2020.

[18] Fouladi, E., Baghaee, H. R., Bagheri, M., \& Gharehpetian, G. , “ Power Management of Microgrids including PHEVs based on Maximum Employment of Renewable Energy Resources", IEEE Transactions on Industry Applications, 2020. 